\title{
Intuitionistic fuzzy based regular and normal spaces
}

\author{
Tamanna Tasnim Prova ${ }^{1, *}$ and Md. Sahadat Hossain ${ }^{2}$ \\ ${ }^{1}$ Department of Mathematics, Rajshahi University of Engineering and Technology \\ Rajshahi-6204, Bangladesh \\ e-mail: tprova.ku@gmail.com \\ ${ }^{2}$ Department of Mathematics, University of Rajshahi \\ Rajshahi-6205, Bangladesh \\ e-mail: sahadateru.ac.bd \\ * Corresponding author
}

Received: 22 October 2020

Revised: 4 December 2020 Accepted: 15 December 2020

\begin{abstract}
In this paper, we define the notion of intuitionistic fuzzy based regular and normal spaces. We also study that classical regular and normal spaces are also intuitionistic fuzzy based regular and normal spaces but the converses are not true in general. This notion opens up a new conception of generalization of classical regular and normal spaces. The hereditary and topological properties of intuitionistic fuzzy based regular and normal spaces have been also investigated. Moreover, by setting some examples we show that every intuitionistic fuzzy based regular space as well as intuitionistic fuzzy based normal space need not be $T_{1}$ spaces. Finally, it is shown that under some conditions the images and homeomorphic images are preserved in intuitionistic fuzzy based regular and normal spaces.
\end{abstract}

Keywords: Intuitionistic fuzzy based set, Intuitionistic fuzzy based topological space, Hereditary, Regular space, Normal space.

2010 Mathematics Subject Classification: 54A05, 54C50. 


\section{Introduction}

Ever since the invention of topological spaces, many researchers have been paying remarkable contributions in this field. By investigating different properties on classical topological spaces, they also added new notions for its generalization. After the introduction of the fuzzy set by Zadeh [28] the classical topological space is directed to a new dimension namely "Fuzzy Topological Spaces" which is defined by Chang [6]. After that, Atanassov [4, 5] introduced the notion of intuitionistic fuzzy set which is a generalization of fuzzy set. Later, Coker et al. [7-10] defined intuitionistic fuzzy topological spaces, intuitionistic sets and intuitionistic topological spaces. From the onwards, Coker and S. Bayhan [9, 10], Singh and Srivastava [24], S. J. Lee and E. P. Lee [14], Saadati and Park [20], Estiaq Ahmed et al. [1, 2], Islam et al. [12] initiated different studies on intuitionistic fuzzy topological spaces by using intuitionistic fuzzy sets. Besides, many researchers have been working for the generalization of topological spaces and intuitionistic fuzzy based topological spaces [3, 13, 15-20, 24-28]. In 2018, S. Selvanayaki et al. [21-23] studied on normal and weak regular space in intuitionistic fuzzy based topological space. In this paper, we studied various properties of intuitionistic fuzzy based regular and normal spaces, the relation between them and their relations with intuitionistic fuzzy based $T_{1}$ space.

\section{Notation and preliminaries}

In this paper $X$ is noted as an non-empty set, $T$ is topology, $(X, T)$ is topological space, $\widetilde{\tau}$ is an intuitionistic fuzzy based topology, $(X, \widetilde{\mathrm{T}})$ is intuitionistic fuzzy based topological space, $G=\left(G_{1}, G_{2}\right)$ and $H=\left(H_{1}, H_{2}\right)$ are intuitionistic fuzzy based sets, $f$ is a function, $\widetilde{\top}_{A}$ is an intuitionistic fuzzy based relative topology on $A$ where $A \subseteq X, \emptyset=(\varnothing, X)$ and $X=(X, \emptyset)$ are also noted as intuitionistic fuzzy based sets.

Definition 2.1 [9]. Suppose $X$ is a non-empty set. An intuitionistic fuzzy based set $A$ and $X$ is an object having the form $A=\left(X, A_{1}, A_{2}\right)$ where $A_{1}$ and $A_{2}$ are subsets of $X$ satisfying $A_{1} \cap A_{2}=\emptyset$. The set $A_{1}$ is called the set of member of $A$ while $A_{2}$ is called the set of non-member of $A$. In this paper, we use the simpler notation $A=\left(A_{1}, A_{2}\right)$ instead of $A=\left(X, A_{1}, A_{2}\right)$ for an intuitionistic fuzzy based set.

Remark 2.1 [9]. Every subset $A$ of a nonempty set $X$ may obviously be regarded as an intuitionistic fuzzy based set having the form $A=\left(A, A^{c}\right)$ where $A^{c}=X / A$.

Definition 2.2 [9]. Let the intuitionistic fuzzy based sets $A$ and $B$ in $X$ be of the forms $A=\left(A_{1}, A_{2}\right)$ and $B=\left(B_{1}, B_{2}\right)$, respectively. Furthermore, let $\left\{A_{j}, j \in J\right\}$ be an arbitrary family of intuitionistic fuzzy based sets in $X$, where $A_{j}=\left(A_{j}^{(1)}, A_{j}^{(2)}\right)$. Then:

a. $A \subseteq B$ if only if $A_{1} \subseteq B_{1}$ and $A_{2} \supseteq B_{2}$;

b. $A=B$ if and if $A \subseteq B$ and $B \supseteq A$;

c. $\bar{A}=\left(A_{1}, A_{2}\right)$ denotes the complement of $A$;

d. $\cap A_{j}=\left(\cap A_{j}^{(1)}, \cup A_{j}^{(2)}\right)$; 

e. $\cup A_{j}=\left(\cup A_{j}^{(1)}, \cap A_{j}^{(2)}\right)$;
f. $\emptyset_{\sim}=(\varnothing, X)$ and $X_{\sim}=(X, \varnothing)$.

Definition 2.3 [4]. Let $X$ be a non-empty set. A family $\widetilde{\top}$ of intuitionistic fuzzy based sets in $X$ is called an intuitionistic fuzzy based topology on $X$ if the following conditions hold.
a. $\emptyset_{\sim}, X_{\sim} \in \widetilde{\top}$;
b. $A \cap B \in \widetilde{\mathrm{T}}$ for all $A, B \in \widetilde{\mathrm{T}}$;
c. $\cup A_{j} \in \widetilde{\mathrm{T}}$ for any arbitrary family $\left\{A_{j} \in \widetilde{\mathrm{T}}, j \in J\right\}$.

The pair $(X, \widetilde{\tau})$ is called an intuitionistic fuzzy based topological space (IFTS, in short), members of $\widetilde{\tau}$ are called intuitionistic fuzzy based open sets (IFOS, in short) in $X$ and their complements are called intuitionistic fuzzy based closed sets (IFCS, in short) in $X$.

Definition 2.4 [1]. Let $(X, \widetilde{\mathrm{T}})$ and $(Y, \delta)$ be IFTSs. A function $f: X \rightarrow Y$ is called continuous if $f^{-1}(B) \in$ T for all $B \in \delta$ and $f$ is called open if $f(A) \in \delta$ for all $A \in \widetilde{T}$.

Definition 2.5 [4]. An intuitionistic fuzzy based topological space $(X, \breve{\tau})$ is called $T_{1}$ if for all $x, y \in X$ with $x \neq y$ there exist intuitionistic fuzzy based sets $A=\left(A_{1}, A_{2}\right), B=\left(B_{1}, B_{2}\right) \in \widetilde{\mathrm{T}}$, such that $x \in A_{1}, y \notin A_{1}$ and $y \in B_{1}, x \notin B_{1}$.

Definition 2.6 [9]. Let $X$ and $Y$ be two nonempty sets and $f: X \rightarrow Y$ be a function. If $A=\left(A_{1}, A_{2}\right) \subseteq X$, then $f(A)$ is defined as $f(A)=\left(f\left(A_{1}\right), f\left(A_{2}\right)\right)$ and if $=\left(B_{1}, B_{2}\right) \subseteq Y$, then $f^{-1}(B)$ is defined as $f^{-1}(B)=\left(f^{-1}\left(B_{1}\right), f^{-1}\left(B_{2}\right)\right)$.

\section{Regular and normal spaces in intuitionistic fuzzy based topological spaces}

In this section, we will define the notions of intuitionistic fuzzy based subspace, intuitionistic fuzzy based regular and normal spaces and examine their properties. By setting some examples we will show that intuitionistic fuzzy based regular and normal spaces are more general than classical regular and normal spaces. We will also find the relation between them. Furthermore, it will be examined here that every intuitionistic fuzzy based regular and normal space need not be intuitionistic fuzzy based $T_{1}$ space.

Definition 3.1. Let $(X, \widetilde{\tau})$ be an intuitionistic fuzzy based topological space and $A \subseteq X$. If $\widetilde{\tau}_{A}$ is a topology generated by $\{G \cap(A, \varnothing): G \in \widetilde{\mathrm{T}}\}$, then $\widetilde{\top}_{A}$ is also an intuitionistic fuzzy based topology on $A$ and the space $\left(A, \widetilde{\top}_{A}\right)$ is a subspace of $(X, \mathrm{~T})$.

Definition 3.2. An intuitionistic fuzzy based space $(X, \widetilde{\mathrm{T}})$ is regular if for any $p \in X$ and $G=\left(G_{1}, G_{2}\right) \in \widetilde{\mathrm{T}}$ with $p \notin G_{2}$ there exist disjoint open sets $A=\left(A_{1}, A_{2}\right)$ and $B=\left(B_{1}, B_{2}\right)$, such that $G^{c}=\left(G_{2}, G_{1}\right) \subset \mathrm{A}$ and $p \in B_{1}$.

Definition 3.3. An intuitionistic fuzzy based space $(X, \widetilde{\top})$ is normal if $G=\left(G_{1}, G_{2}\right) \in \widetilde{\top}$, $H=\left(H_{1}, H_{2}\right) \in \widetilde{\mathrm{T}}$ with $G_{2} \cap H_{2}=\varnothing$ then there exist disjoint open sets $A=\left(A_{1}, A_{2}\right)$ and $B=\left(B_{1}, B_{2}\right)$ such that $G^{c}=\left(G_{2}, G_{1}\right) \subset \mathrm{A}$ and $H^{c}=\left(H_{2}, H_{1}\right) \subset \mathrm{B}$. 
Theorem 3.1. Every classical regular space can be represented as an intuitionistic fuzzy based regular space but the converse is not true.

Proof. Let $(X, \mathrm{~T})$ be a classical regular space. So, according to the axioms of regular space we have, if any $p \in X$ and $G \in \mathrm{T}$ with $p \notin G^{c}$, then there exist two disjoint open sets $A$ and $B$ such that $G^{c} \subset$ A and $p \in B$.

Set $E=\left(G, G^{c}\right), M=\left(A, A^{c}\right), N=\left(B, B^{c}\right)$, then it is clear that $p \in X$ and $E=\left(G, G^{c}\right) \in \widetilde{\top}$, $p \notin G^{c}$, and we get two disjoint sets $M, N \in \widetilde{\mathrm{T}}$ (since $A, B$ disjoint) such that $E^{c}=\left(G^{c}, G\right) \subset$ $M=\left(A, A^{c}\right)$ (as $\left.G^{c} \subset A\right)$ and $p \in B$. Hence, correspondingly $(X, \widetilde{\mathrm{T}})$ is an intuitionistic fuzzy based regular space.

But the converse is not true. For example, Consider $X=\{a, b, c\}, G=(\{b, c\},\{a\})$, $A=(\{a, c\},\{b\}), B=(\{a, b\},\{c\}), D=(\{a\},\{b\}), E=(\{b\},\{c\}), F=(\{c\},\{a\})$. Let $\widetilde{\tau}$ be an intuitionistic fuzzy based topology generated by $\{G, A, D, E, F\}$. Therefore $(X, \widetilde{\top})$ is an intuitionistic fuzzy based topological space.

Therefore $(X, \widetilde{\top})$ is an intuitionistic fuzzy based topological space generated by $\{G, A, B, D, E, F\}$. Now, choose $b \in X$, with $b \notin\{\mathrm{a}\}$, where $G=(\{b, c\},\{a\}) \in \widetilde{\top}$ there exist disjoint open sets $A$ and $E$ such that $G^{c} \subset \mathrm{A}$ and $b \in E$. Similarly, we can show that this result is true for the points, $a$ and $c$.

$\operatorname{Thus}(X, \breve{\mathrm{T}})$ is an intuitionistic fuzzy based regular space. But this space cannot be represented as classical regular space.

Hence the proof.

Theorem 3.2. Every intuitionistic fuzzy based regular subspace is also regular.

Proof. Let $A \subseteq X$ and $\left(A, \widetilde{\mathrm{T}}_{A}\right)$ be a subspace of the regular space $(X, \widetilde{\mathrm{T}})$. Consider $F=\left(F_{2}, F_{1}\right)$ is $\widetilde{\mathrm{T}}_{A}$-closed set in $A$ then $F^{c}=\left(F_{1}, F_{2}\right)$ is $\widetilde{\mathrm{T}}_{A}$-open set in $A$. Consider $p \in A$ with $\mathrm{p} \notin F_{2}$. Since $F^{c}=\left(F_{1}, F_{2}\right) \in \widetilde{\mathrm{T}}_{A}$, then by the definition of subspace $\exists E=\left(E_{1}, E_{2}\right) \in \widetilde{\mathrm{T}}$ such that $F^{c}=E \cap(A, \varnothing) \Rightarrow\left(F_{1}, F_{2}\right)=\left(E_{1}, E_{2}\right) \cap(A, \emptyset)=\left(E_{1} \cap A, E_{2}\right)$, i.e., $F_{1}=E_{1} \cap A$ and $F_{2}=E_{2}$.

Since $\mathrm{p} \notin F_{2}$ then $\mathrm{p} \notin E_{2}$. So we have a $\breve{\mathrm{T}}$ - closed set $E^{c}=\left(E_{2}, E_{1}\right)$ such that $\mathrm{p} \notin E_{2}$. Since $(X, \widetilde{\mathrm{T}})$ is an intuitionistic fuzzy based regular space then there exist two disjoint open sets $G=\left(G_{1}, G_{2}\right)$ and $H=\left(H_{1}, H_{2}\right)$ such that $E^{c} \subset G$ and $\mathrm{p} \in H_{1}$. Now, $E^{c}=\left(E_{2}, E_{1}\right) \subset\left(G_{1}, G_{2}\right)$ implies that $E_{2} \subset G_{1}$ and $E_{1} \supset G_{2}$. Furthermore, $E_{2} \subset G_{1} \Rightarrow F_{2} \subset G_{1} \Rightarrow F_{2} \cap A \subset G_{1} \cap A \Rightarrow$ $F_{2}=F_{2} \cap A \subset G_{1} \cap A$. Again, $E_{1} \supset G_{2} \Rightarrow E_{1} \cap A \supset G_{2} \cap A \Rightarrow F_{1} \supset G_{2} \cap A$.

Therefore, $\left(F_{2}, F_{1}\right) \subset\left(G_{1} \cap A, G_{2} \cap A\right)=G \cap A$, as $F_{1} \cap F_{2}=\emptyset$ and $G_{1} \cap G_{2}=\emptyset$.

Again, since $p \in A$ and $p \in H_{1}$ then $\mathrm{p} \in H_{1} \cap A$. Further, since $G$ and $H$ are disjoint then $G \cap A$ and $H \cap A$ are also disjoint.

Finally, we have, $p \in A$ with $p \notin F_{2}$ there exist two disjoint open sets $G \cap A \in \widetilde{\mathrm{T}}_{\boldsymbol{A}}$ and $H \cap A \in \widetilde{\mathrm{T}}_{\boldsymbol{A}}$ such that $F \subset G \cap A$ and $\mathrm{p} \in H_{1} \cap A$. Thus $\left(A, \widetilde{\mathrm{T}}_{A}\right)$ is an intuitionistic fuzzy based regular space.

Theorem 3.3. Let $(X, \widetilde{\mathrm{T}})$ and $(Y, \check{\delta})$ be two intuitionistic fuzzy based topological spaces and $f: X \rightarrow Y$ be one-one, onto, continuous and open map. If $(X, \widetilde{\mathrm{T}})$ is intuitionistic fuzzy based regular space then $(Y, \check{\delta})$ is also intuitionistic fuzzy based regular. 
Proof. Let $(X, \widetilde{\top})$ and $(Y, \check{\delta})$ be two intuitionistic fuzzy based topological spaces and $f: X \rightarrow Y$ be a one-one, onto and open map. Let $(X, \widetilde{\tau})$ is intuitionistic fuzzy based regular space, we shall show that $(Y, \check{\delta})$ is also intuitionistic fuzzy based regular.

Let $p \in Y$ with $p \notin G_{2}$, where $G=\left(G_{1}, G_{2}\right) \in \check{\delta}$, then there exists an $a \in X$ such that $f(a)=p$ as $f$ is onto. Again, $p$ is unique for $a$ as $f$ is one-one. Furthermore, $G \in \check{\delta} \Rightarrow f^{-1}(G) \in \breve{T}$ as $f$ is continuous. And $p \notin G_{2} \Rightarrow a=f^{-1}(p) \notin f^{-1}\left(G_{2}\right)$. Since $(X, \widetilde{\mathrm{T}})$ is intuitionistic fuzzy based regular space, so for any $a \in X$ with $a \notin f^{-1}\left(G_{2}\right)$ where $f^{-1}(G)=\left(f^{-1}\left(G_{1}\right)\right.$, $\left.f^{-1}\left(G_{2}\right)\right) \in \widetilde{\top}$, then there exist two disjoint open sets $A=\left(A_{1}, A_{2}\right), B=\left(B_{1}, B_{2}\right) \in \widetilde{\top}$ such that $f^{-1}\left(G^{c}\right) \subset$ A and $a \in B_{1}$.

Since $A, B \in \breve{\mathrm{T}} \Rightarrow f(A), f(B) \in \check{\delta}$ as $f$ is open. Now we have, $f(A)=\left(f\left(A_{1}\right), f\left(A_{2}\right)\right)$, $f(B)=\left(f\left(B_{1}\right), f\left(B_{2}\right)\right)$. Furthermore, since $a \in B_{1} \Rightarrow f(a)=p \in f\left(B_{1}\right)$ and $f^{-1}\left(G^{c}\right) \subset \mathrm{A}$ $\Rightarrow f f^{-1}\left(G^{c}\right) \subset f(\mathrm{~A}) \Rightarrow G^{c} \subset f(\mathrm{~A})$. Furthermore, $f(\mathrm{~A}), f(\mathrm{~B})$ are disjoint as $A, B$ are disjoint.

Finally, we get, for any $p \in Y$ with $p \notin G_{2}$ there exist two disjoint open sets $f(A), f(B) \in \check{\delta}$ such that $f\left(G^{c}\right) \subset f(\mathrm{~A})$ and $p \in f\left(B_{1}\right)$. Thus $(Y, \check{\delta})$ is an intuitionistic fuzzy based regular space.

Theorem 3.4. Let $(X, \breve{\mathrm{T}})$ and $(Y, \check{\delta})$ be two intuitionistic fuzzy based topological spaces and $f: X \rightarrow Y$ be one-one, onto, continuous and open map. If $(Y, \check{\delta})$ is intuitionistic fuzzy based regular space then $(X, \widetilde{\tau})$ is also intuitionistic fuzzy based regular.

Proof. Let $(X, \breve{\top})$ and $(Y, \check{\delta})$ be two intuitionistic fuzzy based topological spaces and $f: X \rightarrow Y$ be one-one, onto and open map. Let $(Y, \check{\delta})$ be intuitionistic fuzzy based regular space, we shall show that $(X, \widetilde{\mathrm{T}})$ is also intuitionistic fuzzy based regular.

Let $p \in X$ with $p \notin G_{2}$, where $G=\left(G_{1}, G_{2}\right) \in \widetilde{T}$ then there exists an $a \in Y$ such that $a=f(p)$, for unique $p$ as $f$ is one-one. Furthermore, $G \in \breve{\tau} \Rightarrow f(G) \in \check{\delta}$ as $f$ is open. And $p \notin G_{2} \Rightarrow a=f(p) \notin f\left(G_{2}\right)$. Now, since $(Y, \check{\delta})$ is intuitionistic fuzzy based regular space, so for any $a \in Y$ with $a \notin f\left(G_{2}\right)$, where $G=\left(G_{1}, G_{2}\right) \in \check{\delta}$ then there exist two disjoint open sets $A=\left(A_{1}, A_{2}\right), B=\left(B_{1}, B_{2}\right) \in \check{\delta}$ such that $f\left(G^{c}\right) \subset \mathrm{A}$ and $a \in B_{1}$. Again since $\mathrm{A}, B \in \check{\delta} \Rightarrow$ $f^{-1}(A), f^{-1}(B) \in \widetilde{\top}, \quad$ as $f$ is continuous. Now we have, $f^{-1}(A)=$ $\left(f^{-1}\left(A_{1}\right), f^{-1}\left(A_{2}\right)\right), f^{-1}(B)=\left(f^{-1}\left(B_{1}\right), f^{-1}\left(B_{2}\right)\right)$.

Furthermore, $f(p) \in B_{1} \Rightarrow f^{-1} f(p) \in f^{-1}\left(B_{1}\right) \Rightarrow p \in f^{-1}\left(B_{1}\right)$. And $f\left(G^{c}\right) \subset \mathrm{A} \Rightarrow$ $f^{-1} f\left(G^{c}\right) \subset f^{-1}(\mathrm{~A}) \Rightarrow G^{c} \subset f^{-1}(\mathrm{~A})$.

Furthermore, $f^{-1}(\mathrm{~A}), f^{-1}(\mathrm{~B})$ are disjoint as $A, B$ are disjoint.

Finally, we get, for any $p \in X$ with $p \notin G_{2}$ there exist two disjoint open sets $f^{-1}(\mathrm{~A}), f^{-1}(\mathrm{~B}) \in \widetilde{\mathrm{T}}$ such that $G^{c} \subset f^{-1}(\mathrm{~A})$ and $p \in f^{-1}\left(B_{1}\right)$. Thus $(X, \widetilde{\mathrm{T}})$ is an intuitionistic fuzzy based regular space.

Theorem 3.5. Intuitionistic fuzzy based regular space need not be intuitionistic fuzzy based normal space.

Proof. An intuitionistic fuzzy based regular space need not be an intuitionistic fuzzy based normal space. For example, consider $X=\{a, b, c\}, G=(\{b, c\},\{a\}), A=(\{a, c\},\{b\})$, 
$B=(\{a, b\},\{c\}), D=(\{a\},\{b\}), E=(\{b\},\{c\}), F=(\{c\},\{a\})$. Let $\widetilde{\tau}$ be an intuitionistic fuzzy based topology generated by $\{G, A, D, E, F\}$. Therefore $(X, \widetilde{\mathrm{T}})$ is an intuitionistic fuzzy based regular space (see Theorem 3.1). But $(X, \widetilde{\mathrm{T}})$ is not an intuitionistic fuzzy based normal space. Because for two disjoint closed subsets $G^{c}$ and $B^{c}$ there are no disjoint open sets so that $G^{c}$ and $B^{c}$ hold the normal space's axioms. Hence the proof.

Theorem 3.6. Every intuitionistic fuzzy based regular space need not be intuitionistic fuzzy based $T_{1}$ space.

Proof. An intuitionistic fuzzy based regular space need not be an intuitionistic fuzzy based $T_{1}$ space. For example, consider $X=\{a, b, c, p\}, G=(\{b, c\},\{a\}), \mathrm{A}=(\{a, c\},\{b\}), B=(\{a, b\},\{c\})$, $D=(\{a\},\{b\}), E=(\{b\},\{c\}), F=(\{c\},\{a\}), M=(\{b, p\},\{a, c\}), \mathrm{N}=(\{b, p\},\{a\})$. Let $\widetilde{T}$ be an intuitionistic fuzzy based topology generated by $\{G, A, D, E, F, M, N\}$. Therefore, $(X, \widetilde{\tau})$ is an intuitionistic fuzzy based regular space. Now, choose $b \in X$, with $b \notin\{\mathrm{a}\}$, where $G=(\{b, c\},\{a\}) \in \widetilde{\tau}$ there exist disjoint open sets $\mathrm{A}$ and $\mathrm{E}$ such that $G^{c} \subset \mathrm{A}$ and $b \in E$. Similarly we can show that this result is true for the points, $a, c$ and $p$. But the space is not an intuitionistic fuzzy based $T_{1}$ space. Because for the points $b, p$ there are no open sets such that $b$ and $p$ can belong to the sets separately.

Theorem 3.7. Every classical normal space can be represented as an intuitionistic fuzzy based normal space but the converse is not true.

Proof. Let $(X, \mathrm{~T})$ be classical normal space. So according to the axioms of normal space we have if for any $G, H \in \widetilde{\mathrm{T}}$ with $G^{c} \cap H^{c}=\varnothing$, then there exist disjoint open sets A and B such that $G^{c} \subset \mathrm{A}, H^{c} \subset \mathrm{B}$.

Set $E=\left(G, G^{c}\right), F=\left(H, H^{c}\right), M=\left(A, A^{c}\right), N=\left(B, B^{c}\right)$, then it is clear that $E^{c}, F^{c}$ are disjoint (since $G^{c}, H^{c}$ are disjoint), and we get two disjoint sets $M, N \in \widetilde{\mathrm{T}}$ (since $A, B$ disjoint) such that $E^{c}=\left(G^{c}, G\right) \subset M=\left(A, A^{c}\right) \quad\left(\right.$ as $\left.G^{c} \subset A\right)$ and $F^{c}=\left(H^{c}, H\right) \subset N=\left(B, B^{c}\right)$ (as $H^{c} \subset B$ ). Hence, corresponding $(X, \widetilde{\top})$ is intuitionistic fuzzy based normal space. But the converse is not true. For example, consider $X=\{a, b, c, d, e, f, p, q\}, A=(\{c, d, p\},\{a, b\})$, $B=(\{a, b, f\},\{c, d\}), G=(\{a, b, e\},\{c, d\}), H=(\{c, d, q\},\{a\})$. Let $\widetilde{\mathrm{T}}$ be an intuitionistic fuzzy based topology generated by $\{A, B, G, H\}$. Therefore, $(X, \widetilde{\mathrm{T}})$ is an intuitionistic fuzzy based topological space. Thus $(X, \widetilde{\mathrm{T}})$ is also intuitionistic fuzzy based normal space because, for any two disjoint closed sets $A^{c}=(\{a, b\},\{c, d, p\})$ and $B^{c}=(\{c, d\},\{a, b, f\})$ there exist two disjoint open sets $G=(\{a, b, e\},\{c, d\}), \quad H=(\{c, d, q\},\{a\}) \in \widetilde{\mathrm{T}}$ such that $A^{c} \subset \mathrm{G}$ and $B^{c} \subset \mathrm{H}$. This result is true for any other pair of disjoint closed sets. Thus $(X, \widetilde{\mathrm{T}})$ is an intuitionistic fuzzy based normal space. But this space cannot be represented as classical normal space.

Theorem 3.8. Let $(X, \widetilde{\top})$ be an intuitionistic fuzzy based normal space then $\left(A, \widetilde{\mathrm{T}}_{A}\right)$ is also intuitionistic fuzzy based normal.

Proof. Let $\left(A, \widetilde{\top}_{A}\right)$ be a subspace of the intuitionistic fuzzy based regular space $(X, \widetilde{\mathrm{T}})$. Consider $E=\left(E_{2}, E_{1}\right)$ and $F=\left(F_{2}, F_{1}\right)$ are disjoint $\widetilde{T}_{A^{-}}$closed set in $A$ then $E^{c}=\left(E_{1}, E_{2}\right), F^{c}=\left(F_{1}, F_{2}\right)$ are $\breve{T}_{A}$ - open sets in A. 
By the definition of $\widetilde{\top}_{A}$, we have: $M=\left(M_{1}, M_{2}\right) \in \widetilde{\tau}$ such that $E^{c}=M \cap(A, \emptyset)$

$$
\begin{aligned}
\Rightarrow\left(E_{1}, E_{2}\right) & =\left(M_{1}, M_{2}\right) \cap(A, \varnothing) \\
& =\left(M_{1} \cap A, M_{2}\right) .
\end{aligned}
$$

Therefore, $E_{1}=M_{1} \cap A$ and $E_{2}=M_{2}, N=\left(N_{1}, N_{2}\right) \in \widetilde{\tau}$ such that $F^{c}=N \cap(A, \emptyset)$

$$
\begin{aligned}
\Rightarrow\left(F_{1}, F_{2}\right) & =\left(N_{1}, N_{2}\right) \cap(A, \emptyset) \\
& =\left(N_{1} \cap A, N_{2}\right),
\end{aligned}
$$

i.e., $F_{1}=N_{1} \cap A$ and $F_{2}=N_{2}$.

Now $\quad M^{c} \cap N^{c}=\left(M_{2}, M_{1}\right) \cap\left(N_{2}, N_{1}\right)=\left(M_{2} \cap N_{2}, M_{1} \cup N_{1}\right)=\left(E_{2} \cap F_{2}, M_{1} \cup N_{1}\right)=$ $\left(\phi, M_{1} \cup N_{1}\right)$ as $E$ and $F$ are disjoint, so $M^{c}$ and $N^{c}$ are disjoint.

We have $M^{c}$ and $N^{c}$ disjoint $\widetilde{\tau}$-closed sets. Since $(X, \widetilde{\mathrm{T}})$ is an intuitionistic fuzzy based normal space then there exist two disjoint $\widetilde{\top}$-open sets $G=\left(G_{1}, G_{2}\right)$ and $H=\left(H_{1}, H_{2}\right)$ such that $M^{c} \subset \mathrm{G}$ and $N^{c} \subset$ H. Now, $M^{c}=\left(M_{2}, M_{1}\right) \subset\left(G_{1}, G_{2}\right)$ implies that $M_{2} \subset G_{1}$ and $M_{1} \supset G_{2}$.

Furthermore, $M_{2} \subset G_{1}$

$$
\begin{aligned}
& \Rightarrow E_{2} \subset G_{1} \\
& \Rightarrow E_{2} \cap A \subset G_{1} \cap A \\
& \Rightarrow E_{2}=E_{2} \cap A \subset G_{1} \cap A .
\end{aligned}
$$

Again, $M_{1} \supset G_{2}$

$$
\begin{aligned}
& \Rightarrow M_{1} \cap A \supset G_{2} \cap A \\
& \Rightarrow E_{1} \supset G_{2} \cap A .
\end{aligned}
$$

Therefore, $\quad\left(E_{2}, E_{1}\right)=E \subset\left(G_{1} \cap A, G_{2} \cap A\right)=G \cap A$, as $E_{1} \cap E_{2}=\emptyset$ and $G_{1} \cap G_{2}=\emptyset$. Similarly, we can show that $\left(F_{2}, F_{1}\right)=F \subset\left(H_{1} \cap A, H_{2} \cap A\right)=H \cap A$, as $F_{1} \cap F_{2}=\varnothing$ and $H_{1} \cap H_{2}=\varnothing$. Further since $G$ and $H$ are disjoint then $G \cap A$ and $H \cap A$ are also disjoint.

Finally, we have, for any two disjoint closed sets $E=\left(E_{2}, E_{1}\right)$ and $F=\left(F_{2}, F_{1}\right)$, where $E^{C}=\left(E_{1}, E_{2}\right) \in \widetilde{\mathrm{T}}_{A}$ and $F^{C}=\left(F_{1}, F_{2}\right) \in \widetilde{\mathrm{T}}_{A}$ then there exist two disjoint open sets $G \cap A \in \widetilde{\mathrm{T}}_{A}$ and $H \cap A \in \widetilde{\mathrm{T}}_{A}$ such that $E \subset G \cap A$ and $\subset H \cap A$. Thus $\left(A, \widetilde{\Upsilon}_{A}\right)$ is an intuitionistic fuzzy based normal space.

Theorem 3.9. Let $(X, \widetilde{\mathrm{T}})$ and $(Y, \check{\delta})$ be two intuitionistic fuzzy based topological spaces and $f: X \rightarrow Y$ be one-one, onto, continuous and open map. If $(X, \widetilde{\mathrm{T}})$ is intuitionistic fuzzy based normal space then $(Y, \check{\delta})$ is also intuitionistic fuzzy based normal.

Proof. Let $(X, \breve{\top})$ and $(Y, \check{\delta})$ be two intuitionistic fuzzy based topological spaces and $f: X \rightarrow Y$ be a one-one, onto and open map. Let $(X, \widetilde{\mathrm{T}})$ is intuitionistic fuzzy based normal space, we shall show that $(Y, \check{\delta})$ is also intuitionistic fuzzy based normal.

Let $G^{c}=\left(G_{2}, G_{1}\right), H^{c}=\left(H_{2}, H_{1}\right)$ are disjoint closed set $\check{\delta}$ closed set, i.e., $G=\left(G_{1}, G_{2}\right)$, $H=\left(H_{1}, H_{2}\right) \in \check{\delta}$ then $f^{-1}(G), f^{-1}(H) \in \widetilde{\top}, f$ is continuous. Now, $f^{-1}(G)=$ $\left(f^{-1}\left(G_{1}\right), f^{-1}\left(G_{2}\right)\right), \quad f^{-1}(H)=\left(f^{-1}\left(H_{1}\right), f^{-1}\left(H_{2}\right)\right), \quad$ i.e., $f^{-1}\left(G^{c}\right)=\left(f^{-1}(G)\right)^{c}=$ $\left(f^{-1}\left(G_{2}\right), f^{-1}\left(G_{1}\right)\right)$ and $f^{-1}\left(H^{c}\right)=\left(f^{-1}(H)\right)^{c}=\left(f^{-1}\left(H_{2}\right), f^{-1}\left(H_{1}\right)\right)$ are disjoint $\widetilde{T}$ closed set (as $G^{c}$ and $H^{c}$ are disjoint, again $f$ is one-one and onto). 
Since $(X, \widetilde{\mathrm{T}})$ is normal space, so for any two disjoint closed sets $f^{-1}\left(G^{c}\right), f^{-1}\left(H^{c}\right)$, where $f^{-1}(G), f^{-1}(H) \in \widetilde{\top}$ there exist two disjoint open sets $A=\left(A_{1}, A_{2}\right), B=\left(B_{1}, B_{2}\right) \in \widetilde{\top}$ such that $f^{-1}\left(G^{c}\right) \subset \mathrm{A}$ and $f^{-1}\left(H^{c}\right) \subset$ B. Since $A, B \in \widetilde{\mathrm{T}} \Rightarrow f(A), f(B) \in \check{\delta}$ as $f$ is open. Now we have, $f(A)=\left(f\left(A_{1}\right), f\left(A_{2}\right)\right), f(B)=\left(f\left(B_{1}\right), f\left(B_{2}\right)\right)$. Furthermore, $f^{-1}\left(G^{c}\right) \subset \mathrm{A}$

$$
\begin{aligned}
& \Rightarrow f f^{-1}\left(G^{c}\right) \subset f(\mathrm{~A}) \\
& \Rightarrow G^{c} \subset f(\mathrm{~A}) .
\end{aligned}
$$

And $f^{-1}\left(H^{c}\right) \subset \mathrm{B}$

$$
\begin{aligned}
& \Rightarrow f f^{-1}\left(H^{c}\right) \subset f(\mathrm{~B}) \\
& \Rightarrow H^{c} \subset f(\mathrm{~B}) .
\end{aligned}
$$

Again, $f(\mathrm{~A}), f(\mathrm{~B})$ are disjoint as $A, B$ are disjoint, $f$ is one-one.

Finally, we get, for any two disjoint closed sets $G^{c}, H^{c}$ where $G=\left(G_{1}, G_{2}\right), H=\left(H_{1}, H_{2}\right) \in$ $\check{\delta}$ there exist two disjoint open sets $f(A) \in \check{\delta}$ and $f(B) \in \check{\delta}$ such that $G^{c} \subset f(A)$ and $H^{c} \subset f(B)$. Thus $(Y, \check{\delta})$ is an intuitionistic fuzzy based normal space.

Theorem 3.10. Let $(X, \widetilde{\top})$ and $(Y, \check{\delta})$ be two intuitionistic fuzzy based topological spaces and $f: X \rightarrow Y$ be one-one, onto, continuous and closed map. If $(Y, \check{\delta})$ is intuitionistic fuzzy based normal space then $(X, \widetilde{\top})$ is also intuitionistic fuzzy based normal.

Proof. Let $(X, \widetilde{\mathrm{T}})$ and $(Y, \check{\delta})$ be two intuitionistic fuzzy based topological spaces and $f: X \rightarrow Y$ be one-one, continuous and open map. Let $(Y, \check{\delta})$ is intuitionistic fuzzy based normal space, we shall show that $(X, \widetilde{\mathrm{T}})$ is also intuitionistic fuzzy based normal.

Let $p, q \in X$ with $p \neq q \Rightarrow f(p), f(q) \in Y$ with $f(p) \neq f(q)$ as $f$ is one-one.

Let $G^{c}=\left(G_{2}, G_{1}\right), H^{c}=\left(H_{2}, H_{1}\right)$ are disjoint $\breve{\tau}$ closed set, i.e., $G=\left(G_{1}, G_{2}\right)$, $H=\left(H_{1}, H_{2}\right) \in \widetilde{\tau}$ then $f\left(G^{c}\right), f\left(H^{c}\right)$, are disjoint $\check{\delta}$-closed set, as $f$ is closed, one-one and onto. Now we have, $f\left(G^{c}\right)=\left(f\left(G_{2}\right), f\left(G_{1}\right)\right), f\left(H^{c}\right)=\left(f\left(H_{2}\right), f\left(H_{1}\right)\right)$. Since $(Y, \check{\delta})$ is normal space, so for any two disjoint $\check{\delta}$ closed sets $f\left(G^{c}\right)$ and $f\left(H^{c}\right)$, there exist two disjoint open sets $A=\left(A_{1}, A_{2}\right), B=\left(B_{1}, B_{2}\right) \in \check{\delta}$ such that $f\left(G^{c}\right) \subset$ A and $f\left(H^{c}\right) \subset$ B.

Again since $A, B \in \check{\delta} \Rightarrow f^{-1}(A), f^{-1}(B) \in \widetilde{T}$, as $f$ is continuous. Now we have, $f^{-1}(A)=$ $\left(f^{-1}\left(A_{1}\right), f^{-1}\left(A_{2}\right)\right), f^{-1}(B)=\left(f^{-1}\left(B_{1}\right), f^{-1}\left(B_{2}\right)\right)$.

Furthermore, $f\left(G^{c}\right) \subset \mathrm{A}$

$$
\begin{aligned}
& \Rightarrow f^{-1} f\left(G^{c}\right) \subset f^{-1}(\mathrm{~A}) \\
& \Rightarrow G^{c} \subset f^{-1}(\mathrm{~A}) .
\end{aligned}
$$

And

$$
\begin{aligned}
& f\left(H^{c}\right) \subset \mathrm{B} \\
\Rightarrow & f^{-1} f\left(H^{c}\right) \subset f^{-1}(\mathrm{~B}) \\
\Rightarrow & H^{c} \subset f^{-1}(\mathrm{~B}) .
\end{aligned}
$$

Again, $f^{-1}(\mathrm{~A})$ and $f^{-1}(\mathrm{~B})$ are disjoint as $A$ and $B$ are disjoint.

Finally, we get, for any two disjoint closed sets $G^{c}, H^{c}$, where $G=\left(G_{1}, G_{2}\right), H=\left(H_{1}, H_{2}\right)$ $\in \widetilde{\mathrm{T}}$ then there exist two disjoint open sets $f^{-1}(\mathrm{~A}) \in \check{\delta}$ and $f^{-1}(\mathrm{~B}) \in \check{\delta}$ such that $G^{c} \subset f^{-1}(\mathrm{~A})$ and $H^{c} \subset f^{-1}(\mathrm{~B})$.

Thus $(X, \widetilde{\mathrm{T}})$ is an intuitionistic fuzzy based normal space. 
Theorem 3.11. Intuitionistic fuzzy based normal space need not be intuitionistic fuzzy based regular space.

Proof. An intuitionistic fuzzy based regular space need not be an intuitionistic fuzzy based normal space. For example, consider $X=\{a, b, c, d, e, f, p, q\}, A=(\{c, d, p\},\{a, b\})$, $B=(\{a, b, f\},\{c, d\}), G=(\{a, b, e\},\{c, d\}), H=(\{c, d, q\},\{a\})$. Let $\widetilde{\top}$ be an intuitionistic fuzzy based topology generated by $\{A, B, G, H\}$. Therefore $(X, \widetilde{\top})$ is an intuitionistic fuzzy based topological space. This $(X, \widetilde{\mathrm{T}})$ is also intuitionistic fuzzy based normal space (see Theorem 3.7). But the space is not an intuitionistic fuzzy based regular space. Because for any point $e \in X$ with $e \notin\{a, b\}$, where $A^{c}=(\{a, b\},\{c, d, p\})$ then there are no two disjoint open sets such that they hold the intuitionistic fuzzy based regular space's axioms. Hence the proof.

Theorem 3.12. Every intuitionistic fuzzy based normal space need not be intuitionistic fuzzy based $T_{1}$ space.

Proof. An intuitionistic fuzzy based normal space need not be an intuitionistic fuzzy based $T_{1}$ space. For example, consider $X=\{a, b, c, d, e, f, p, q\}, A=(\{c, d, p\},\{a, b\})$, $B=(\{a, b, f\},\{c, d\}), G=(\{a, b, e\},\{c, d\}), H=(\{c, d, q\},\{a\})$. Let $\widetilde{\tau}$ be an intuitionistic fuzzy based topology generated by $\{A, B, G, H\}$. Therefore $(X, \widetilde{\top})$ is an intuitionistic fuzzy based topological space. This $(X, \widetilde{T})$ is also intuitionistic fuzzy based normal space (see Theorem 3.7). But the space is not an intuitionistic fuzzy based $T_{1}$ space. Because for the points $a, b$ there are no open sets such that $b$ and $c$ can belong to the sets separately.

\section{Conclusions}

In this paper, we have observed that intuitionistic fuzzy based regular and normal spaces are more general than the classical regular and normal spaces. It is also showed that intuitionistic fuzzy based regular and normal spaces satisfy hereditary and topological property. Moreover, they are preserved under one-one and open mapping. Hopefully this study will open up a new approach for further investigation.

\section{References}

[1] Ahmed, E., Hossain, M.S., \& Ali, D.M. (2014). On intuitionistic fuzzy $T_{0}$ spaces. Journal of Bangladesh Academy of Sciences, 38(2), 197-207.

[2] Ahmed, E., Hossain, M.S., \& Ali, D.M. (2014). On intuitionistic fuzzy $T_{1}$ spaces. Journal of Physical Sciences, 19, 59-66.

[3] Amin, R., Ali, D. M., \& Hossain, M. S. (2015). $T_{1}$ concept in fuzzy bitopological spaces. Italian Journal of Pure and Applied Mathematics, 35, 339-346.

[4] Atanassov, K.T. (1986). Intuitionistic fuzzy sets. Fuzzy Sets and Systems, 20(1), 87-96.

[5] Atanassov, K.T. (2001). On four intuitionistic fuzzy topological operators. Math-ware Soft Comput., 8, 65-70. 
[6] Chang, C.L. (1968). Fuzzy topological space. J. of Mathematical Analysis and Application, 24, 182-90.

[7] Coker, D. (1996). A note on intuitionistic sets and intuitionistic points. Tr. J. of Mathematics, 20(3), 343-351.

[8] Coker, D. (1997). An introduction to intuitionistic fuzzy topological spaces. Fuzzy Sets and Systems, 88(1), 81-89.

[9] Coker, D., \& Bayhan, S. (2001). On separation axioms in intuitionistic topological space. Int. J. of Math. Sci., 27(10), 621-630.

[10] Coker, D., \& Bayhan, S. (2003). On $T_{1}$ and $T_{2}$ separation axioms in intuitionistic fuzzy topologica space, Journal of Fuzzy Mathematics, 11(3), 581-592.

[11] Islam, M.S., Hossain, M.S. \& Asaduzzaman, M. (2018). Level Separation on Intuitionistic fuzzy $T_{1}$ spaces. J. Bangladesh Acad. Sci., 42(1), 73-85.

[12] Islam, R., Hossain, M.S., \& Hoque, M.F. (2020). A study on intuitionistic L-fuzzy $T_{1}$ spaces. Notes on Intuitionistic Fuzzy Sets, 26(3), 33-42.

[13] Kohli, J.K., \& Das, A.K. (2002). New normality axioms and decompositions of normality. Glasnik Mat. 37(57), 163-173.

[14] Lee, S. J., \& Lee, E. P. (2000). The Category of Intuitionistic Fuzzy Topological Space, Bull. Korean Math. Soc., 37(1), 63-76.

[15] Mahbub, M.A., Hossain, M.S., \& Hossain, M.A. (2018). Some properties of compactness in intuitionistic fuzzy topological spaces, Intern. J. Fuzzy Mathematical Archive, 16(2), $39-48$.

[16] Mahbub, M.A., Hossain, M.S., \& Hossain, M.A. (2019). Separation axioms in intuitionistic fuzzy compact topological space. Journal of Fuzzy Set Valued Analysis, 1, 14-23.

[17] Palaniappan, N., Arjuman, K., \& Palanivelrajan, M. (2018). A Study on Intuitionistic L-fuzzy Subrings. Notes on Intuitionistic Fuzzy Sets, 14(3), 5-10.

[18] Roshmi, R., \& Hossain, M. S. (2019). Properties of separation axioms in Bitopological space, J. Bangladesh Acad. Sci., 43(2), 191-195.

[19] Roshmi, R., \& Hossain, M. S. (2020). Regularity and normality in Bitopological space, International Journal of Scientific Research in Mathematical and Statistical Science, 7(1), 21-25.

[20] Saadati R., \& Park, J. H. (2006). On the intuitionistic fuzzy topological spaces. Chaos, Solitons Fractals, 27, 331-344.

[21] Selvanayaki, S., \& Ilango, G. (2015). IGPR continuity and compactness in intuitionistic topological spaces. British Journal of Mathematics \& Computer Science, 11(2), 1-8.

[22] Selvanayaki, S., \& Ilango, G. (2016). Strong and Weak forms of IGPR continuity in intuitionistic topological spaces. International Journal of Pure and Applied Mathematics 106(7), 45-55. 
[23] Selvanayaki, S., \& Ilango, G. (2017). Generators in intuitionistic topological spaces. International Journal of Pure and Applied Mathematics, 116(12), 209-218.

[24] Singh, A.K., \& Srivastava, R. (2012). Separation Axioms in Intuitionistic Fuzzy Topological Spaces. Advances in Fuzzy Systems, Article No. 604396.

[25] Szmidt, E., \& Kacprzyk, J. (2001). Intuitionistic fuzzy sets in some medical applications. Notes on Intuitionistic Fuzzy Sets, 7(4), 58-64.

[26] Szmidt, E., \& Kacprzyk, J. (2004). Medical diagnostic reasoning using a similarity measure for intuitionistic fuzzy sets. Notes on Intuitionistic Fuzzy Sets, 10(4), 61-69.

[27] Younus, J., Yaseen, R., \& Mohammad, A. (2012). Regular and Weak Regular in intuitionistic topological spaces. J. of University of Anbar for Pure Science, 6(3), 1-5.

[28] Zadeh, L.A. (1965). Fuzzy sets, Information and Control, 8(3), 338-353. 